\title{
An Image Denoising Method with Enhancement of the Directional Features Based on Wavelet and SVD Transforms
}

\author{
Min Wang, ${ }^{1}$ Zhen Li, $^{2}$ Xiangjun Duan, ${ }^{3}$ and Wei $\mathrm{Li}^{4}$ \\ ${ }^{1}$ Institute of Meteorology and Oceanography, PLA University of Science and Technology, Nanjing 211101, China \\ ${ }^{2}$ School of Information Engineering, Jiangsu Maritime Institute, Nanjing 211100, China \\ ${ }^{3}$ Nanjing College of Information Technology, Nanjing 210023, China \\ ${ }^{4}$ Department of Control and Systems Engineering, Nanjing University, Nanjing 210093, China \\ Correspondence should be addressed to Xiangjun Duan; dseattle@sina.com
}

Received 26 July 2015; Revised 22 October 2015; Accepted 2 November 2015

Academic Editor: Yann Favennec

Copyright (c) 2015 Min Wang et al. This is an open access article distributed under the Creative Commons Attribution License, which permits unrestricted use, distribution, and reproduction in any medium, provided the original work is properly cited.

\begin{abstract}
This paper proposes an image denoising method, using the wavelet transform and the singular value decomposition (SVD), with the enhancement of the directional features. First, use the single-level discrete 2D wavelet transform to decompose the noised image into the low-frequency image part and the high-frequency parts (the horizontal, vertical, and diagonal parts), with the edge extracted and retained to avoid edge loss. Then, use the SVD to filter the noise of the high-frequency parts with image rotations and the enhancement of the directional features: to filter the diagonal part, one needs first to rotate it 45 degrees and rotate it back after filtering. Finally, reconstruct the image from the low-frequency part and the filtered high-frequency parts by the inverse wavelet transform to get the final denoising image. Experiments show the effectiveness of this method, compared with relevant methods.
\end{abstract}

\section{Introduction}

Denoising is important in image processing. There are many techniques for denoising, such as using the wavelet transform [1-8] and singular value decomposition (SVD) filters [9]. Wavelet transform has multiresolution properties, and the noise of the image is mainly in the high-frequency parts [1-3]; the wavelet threshold method is most widely used for noise filtering, which is effective, but it also removes some useful edge information.

Singular value decomposition (SVD) is generally used as a kind of nonlinear filter, with good numerical robustness [9]. SVD can handle different types of noise, without the prior knowledge about the noise [9]. The directional features of SVD can be used to filter the high-frequency parts of an image, if with proper handling.

This paper proposes a method for image denoising, which utilizes the properties of the wavelet transform and the SVD, with consideration of enhancement of the directional features in the original image.
The method is described as follows. First, use the single-level discrete 2D wavelet transform to decompose the noised image into the low-frequency image part and the high-frequency parts (the horizontal, vertical, and diagonal parts), with the edge extracted and retained to avoid edge loss. Then use the SVD to filter the noise of the highfrequency parts with image rotations and the enhancement of the directional features: to filter the diagonal part, one needs first to rotate it 45 degrees and rotate it back after filtering. Finally, reconstruct the image from the lowfrequency part and the filtered high-frequency parts, by the inverse wavelet transform, to get the final denoising image.

The experiments show that this method is effective in denoising, while it retains the original edge details, compared with the peak signal-to-noise ratio (PSNR) using the relevant methods, and it has better performance than the recent improved wavelet threshold denoising method in [3].

The reminder of the paper is arranged as follows. Section 2 is the principles of wavelet transform and SVD. 


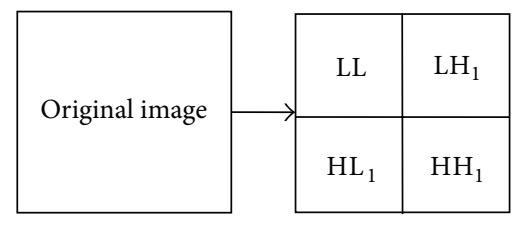

FIGURE 1: Wavelet decomposition: the upper left corner is the smoothing approximation (low-frequency LL). HL: the lower left corner for the vertical part, LH: the upper right corner for the horizontal part, and HH: the lower right corner.

Section 3 is the denoising method. Section 4 is the numerical experiments. Section 5 is the conclusion.

\section{Principles of Wavelet Transform and SVD}

2.1. Wavelet Transform and Threshold Denoising. For a twodimensional image, using the wavelet transform, one gets the four parts: the low-frequency image part and the three highfrequency parts (the horizontal, vertical, and diagonal parts), as shown in Figure 1.

Common threshold functions for denoising are as follows: (1) hard threshold functions and (2) soft threshold functions. He et al. proposed an improved wavelet threshold denoising method [3]: a new index is proposed to measure the interscale correlation of wavelet coefficients as

$$
K(n)=\frac{\max |W(:, n)|-\min |W(:, n)|}{\min |W(:, n)|},
$$

where $W(:, n)$ represents the wavelet coefficients in location point $n . K(n)$ is an index measuring the interscale correlation of wavelet coefficients. Because the universal threshold often has the risk of overkilling the useful information, therefore $K(n) \in[0, r)$, the threshold needs to be shrunk to retain location point $n$, and when $K(n) \in[r,+\infty)$, the threshold of location point $n$ remains invariant. A new threshold determination method considering interscale correlation is presented as

$$
T_{\text {new }}= \begin{cases}0.7 \lambda & K(n) \in[0,0.5 r) \\ 0.8 \lambda & K(n) \in[0.5 r, 0.8 r) \\ 0.9 \lambda & K(n) \in[0.8 r, r) \\ \lambda & K(n) \in[r,+\infty),\end{cases}
$$

where $\lambda$ is universal threshold.

2.2. Introduction of SVD Denoising. The SVD of matrix $A \in$ $R^{l_{1} \times l_{2}}\left(l_{1} \geq l_{2}\right)$ is defined as

$$
A=U S V^{T}
$$

where $U=\left(u_{1}, u_{2}, \ldots, u_{l_{1}}\right) \in R^{l_{1} \times l_{2}}$ and $V=\left(v_{1}, v_{2}, \ldots, v_{l 2}\right) \in$ $R^{l_{1} \times l_{2}}$ are the left singular matrix and the right singular matrix of $A$, the column vector of $U$ and $V$ is the left singular vectors and the right singular vector of $A$. The $\operatorname{rank}$ of $A$ is $\operatorname{rank}(A)=$ $R\left(R \leq l_{2}\right) . S \in R^{l_{1} \times l_{2}}$ is the singular value matrix, with the diagonal elements (the nonzero singular values), as $\lambda_{1} \geq \lambda_{2} \geq$ $\cdots \geq \lambda_{R}>0$. Then $A$ can be expressed as

$$
A=\sum_{i=1}^{R} \lambda_{i} u_{i} v_{i}^{T}
$$

by removing zero singular value of $A$. The singular value reflects the energy distribution of the matrix. We choose the appropriate number of $k$ or singular value threshold $\gamma$ to reconstruct

$$
\widehat{A}=\sum_{i=1}^{k} \lambda_{i} u_{i} v_{i}^{T}, \quad \lambda_{i} \geq \gamma
$$

For an image with only vertical lines, the image signal energy after SVD is concentrated in a few larger singular values. This is the directional characteristics of SVD.

\section{Image Denoising in This Paper}

The algorithm of this paper is as follows (Figure 2):

(1) use the single-level or multilevel discrete 2D wavelet transform to decompose the noised image into the low-frequency image part and the high-frequency parts (the horizontal, vertical, and diagonal parts);

(2) use the edge detection operator to extract the edges (Section 3.1);

(3) use the SVD to filter the noise of the high-frequency parts, with the singular values determined by the PSNR of the image (Section 3.2);

(4) use the SVD to filter the noise of the high-frequency parts with image rotations and the enhancement of the directional features: to filter the diagonal part, one needs to first rotate it 45 degrees and rotate it back after filtering (one illustration is shown in Figure 3);

(5) finally, reconstruct the image from the low-frequency part and the filtered high-frequency parts, using the inverse wavelet transform by the inverse wavelet transform, to get the final denoising image.

3.1. High-Frequency Edge Extraction. Because the highfrequency parts correspond to the edge portion of the original image, we can use the single-direction edge detection operator to extract the edge details: using Sobel edge detection operator to detect the horizontal and vertical direction of the 


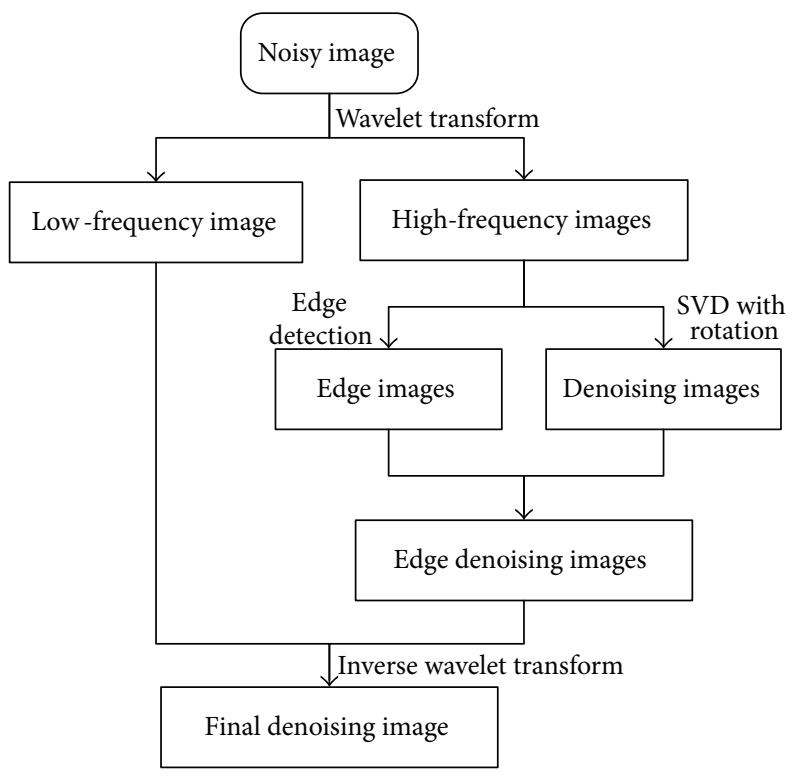

FIGURE 2: Flowchart of this algorithm.

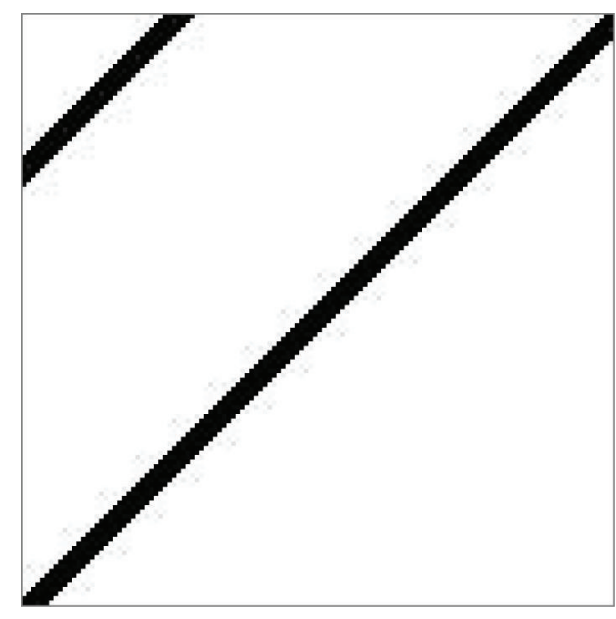

(a) Original image

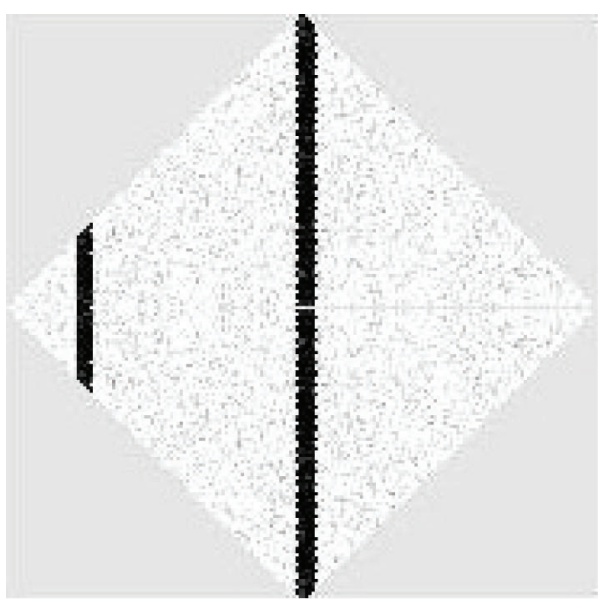

(c) Rotating the image (b)

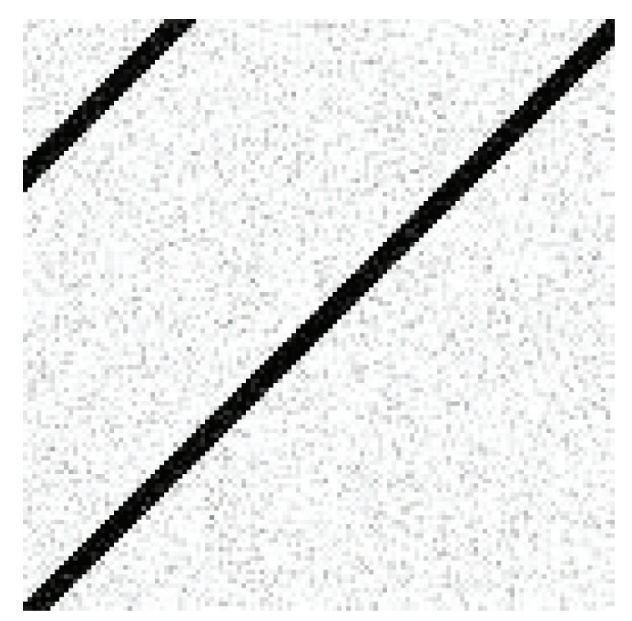

(b) Image (a) with noise

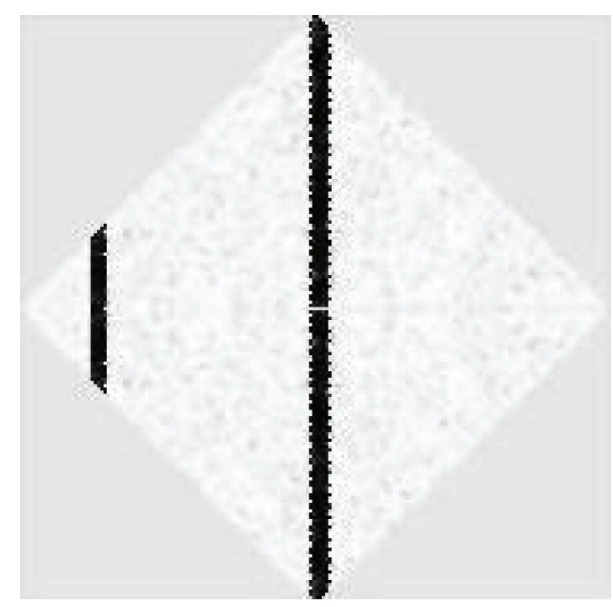

(d) SVD denoising image

Figure 3: SVD denoising image. 


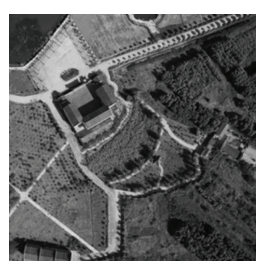

(a)

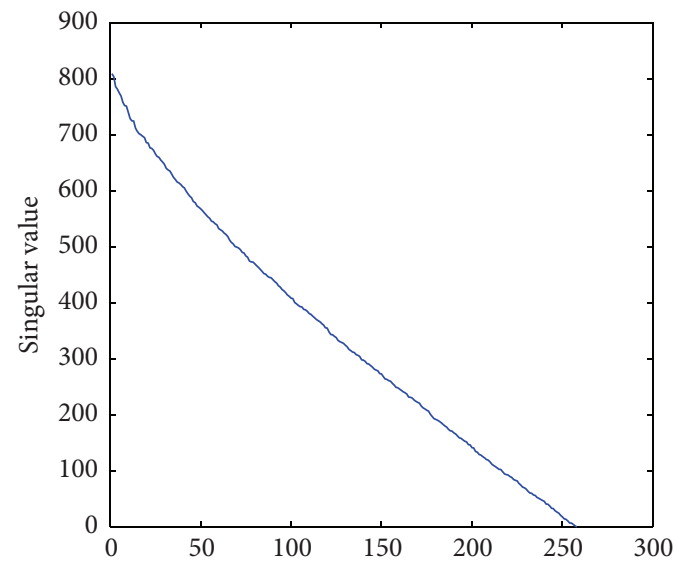

(c)

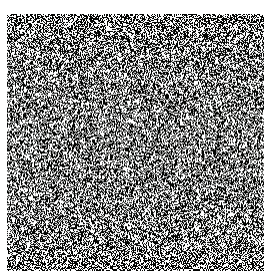

(e-1)

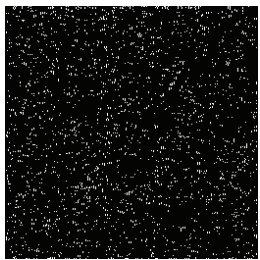

(e-7)

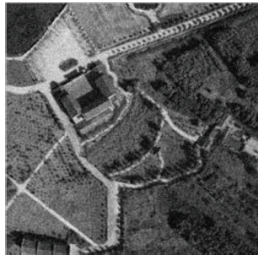

(f-2)

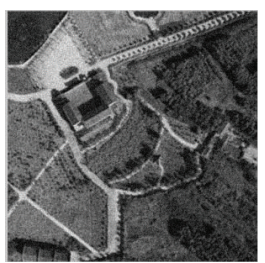

(i-2)

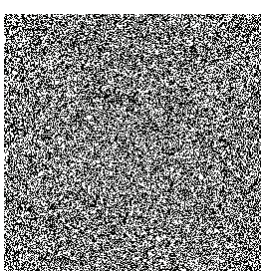

(e-2)

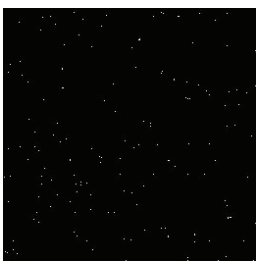

(e-8)

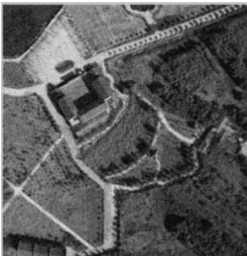

(g-1)

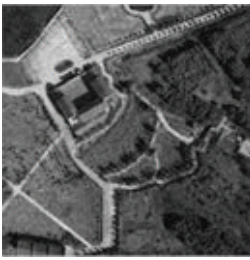

(j-1)

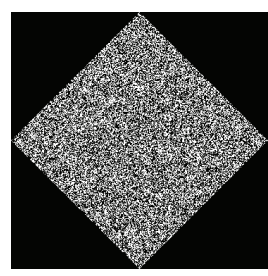

(e-3)

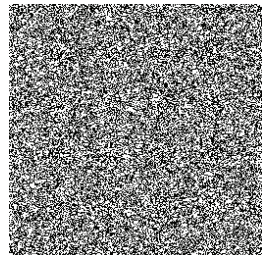

(e-9)

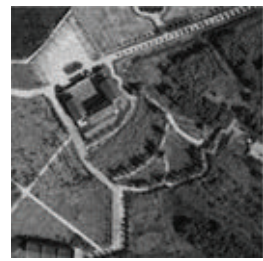

(g-2)

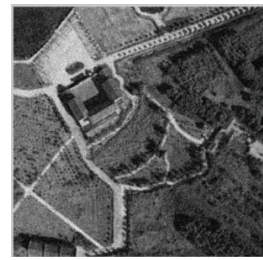

$(j-2)$

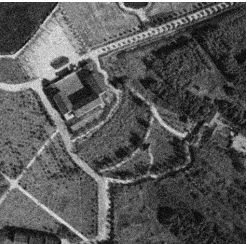

(b)

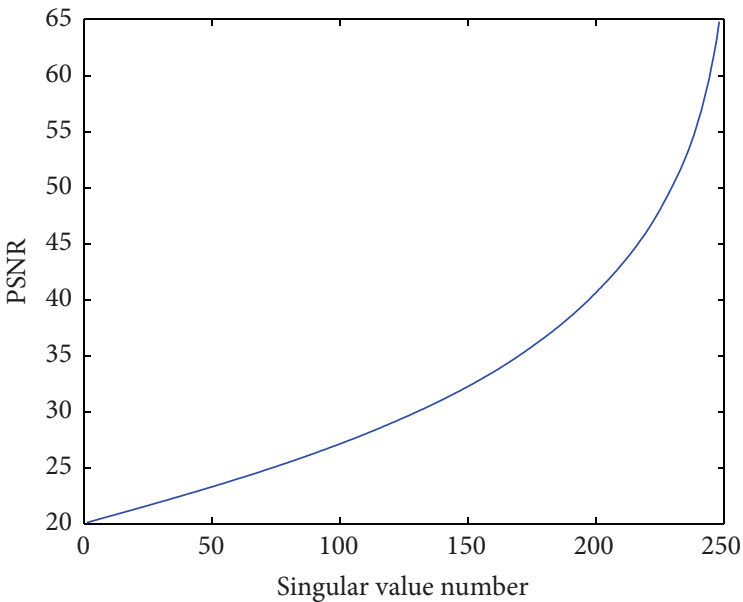

(d)

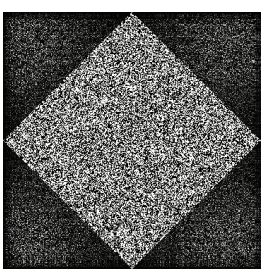

(e-4)

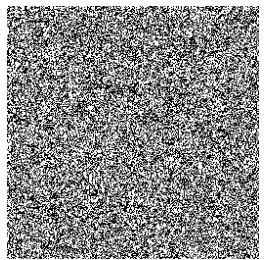

(e-10)

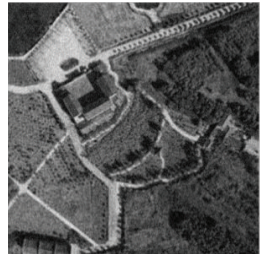

(h-1)

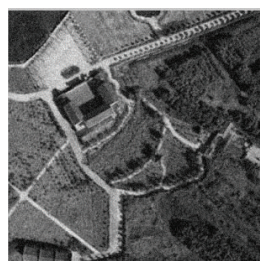

(k)

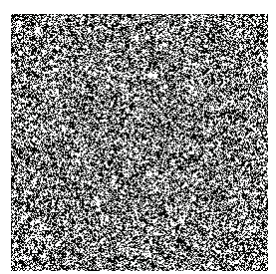

(e-5)

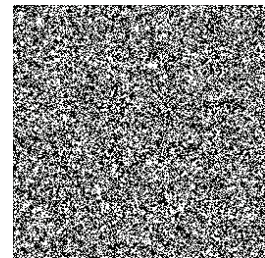

(e-11)

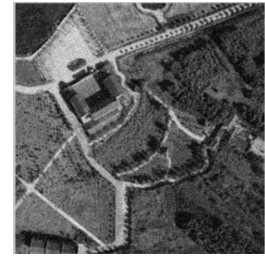

(h-2)

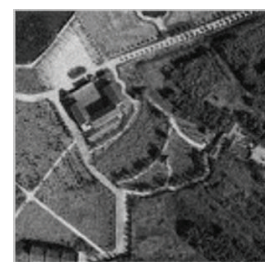

(l)

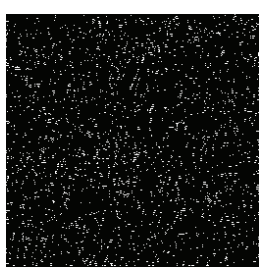

(e-6)

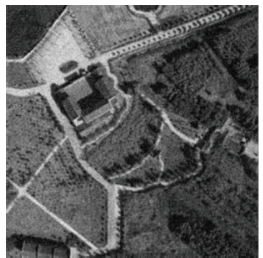

(f-1)

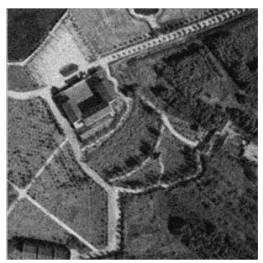

(i-1)

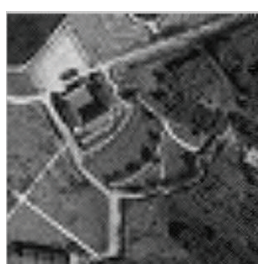

(m)

Figure 4: Continued. 

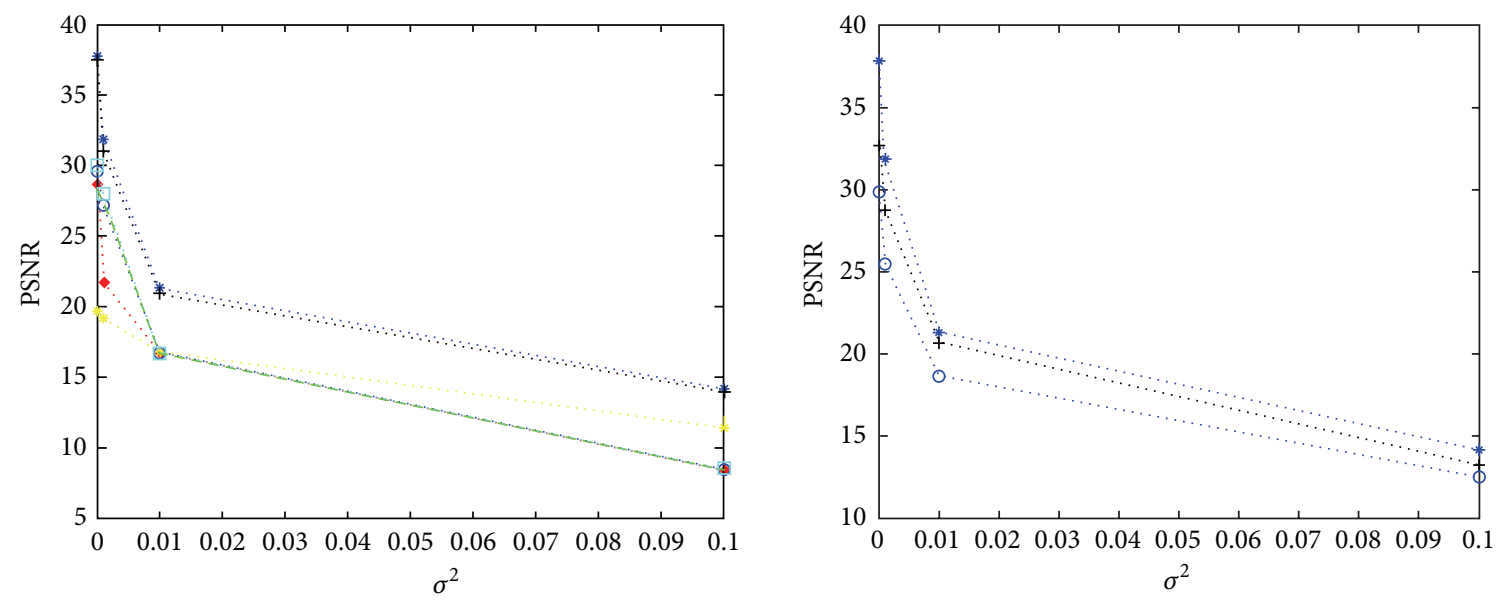

**. The PSNR of this paper (db3 wavelet)

+.. The PSNR of this paper (sym 3 wavelet)

.. . The PSNR of hard thresholding ( $\mathrm{db} 3$ wavelet)

-. The PSNR of hard thresholding (sym3 wavelet)

- - The PSNR of soft thresholding ( $\mathrm{db} 3$ wavelet)

The PSNR of soft thresholding (sym 3 wavelet)

$\square$. The PSNR of universal SVD

(n)

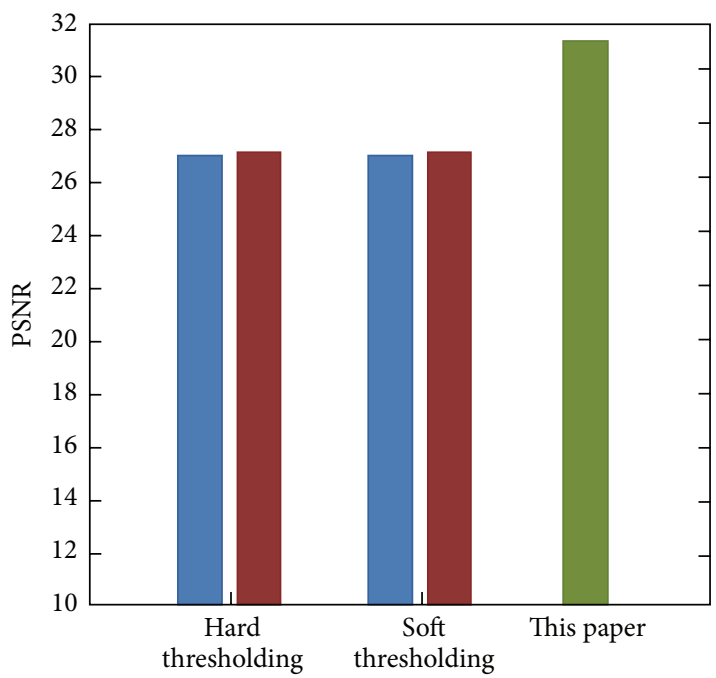

$(\mathrm{p}-1)$

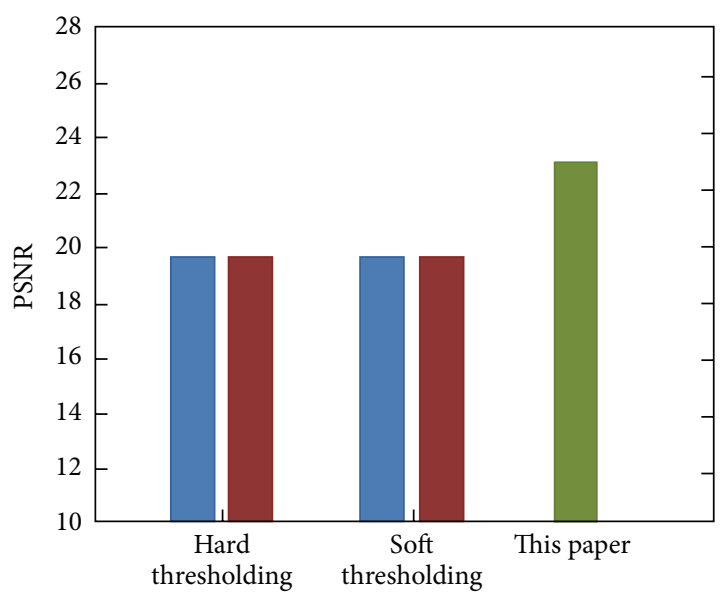

$(\mathrm{q}-1)$
*.. The PSNR of this paper (db3 wavelet, one-layer)

+.. The PSNR of this paper (db3 wavelet, two-layer)

○. . The PSNR of this paper (db3 wavelet, three-layer) (o)

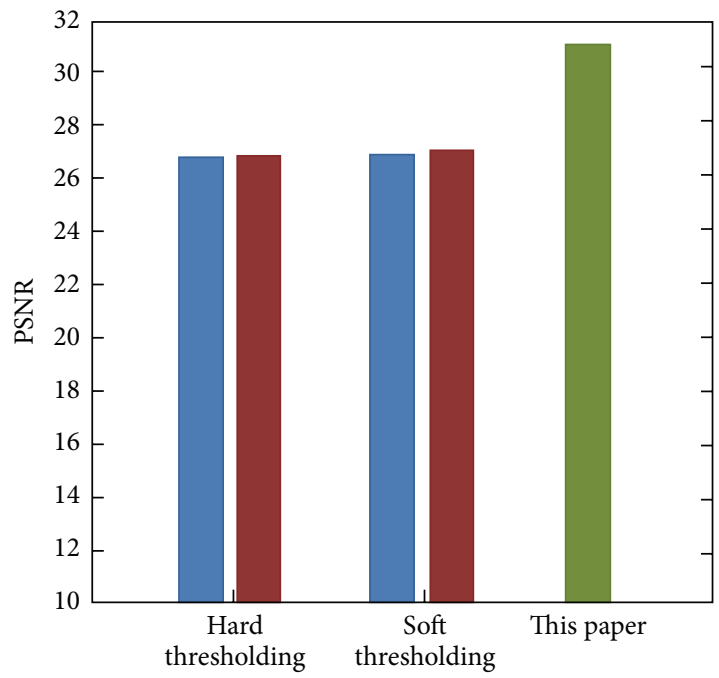

$(\mathrm{p}-2)$

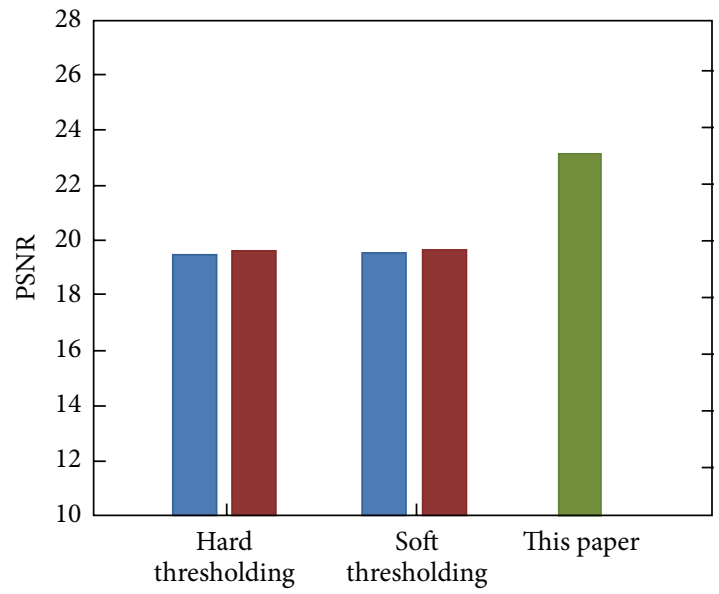

$(\mathrm{q}-2)$

FIgURE 4: Continued. 


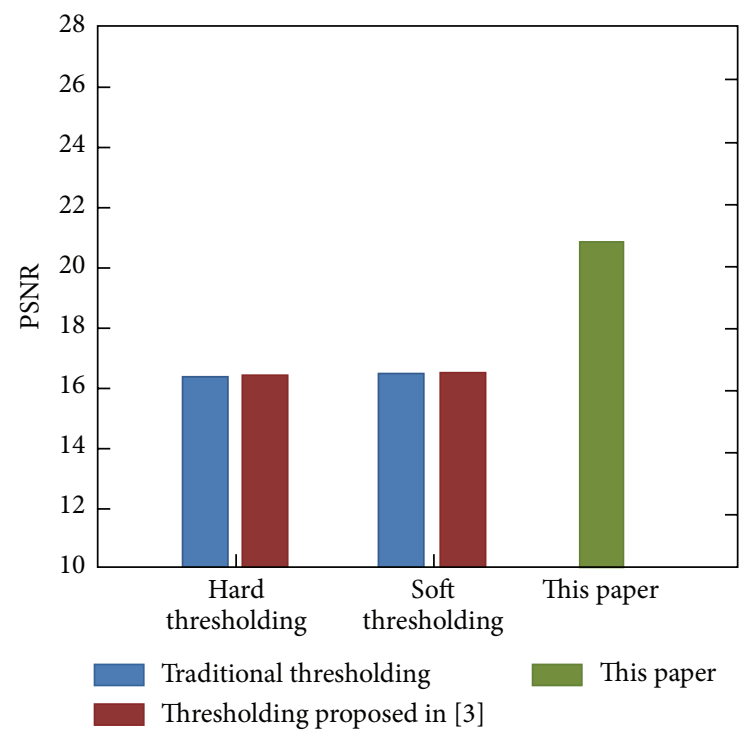

$(\mathrm{r}-1)$

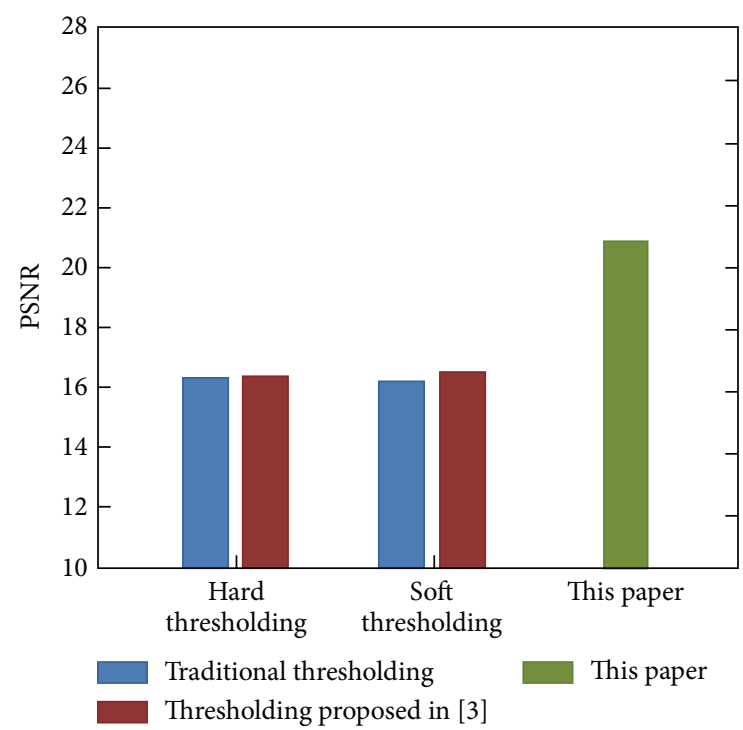

$(\mathrm{r}-2)$

Figure 4: (a) The original image; (b) the noised image $\left(\sigma^{2}=0.01\right)$; (c) the singular value curve of the horizontal high-frequency image; (d) the relation of the singular value number and PSNR; (e-1) the one-layer horizontal high-frequency image using the SVD denoising; (e-2) the one-layer vertical high-frequency image using the SVD denoising; (e-3) the one-layer diagonal high-frequency image with rotation of 45 degrees; (e-4) the denoised image of (e-3) using the SVD denoising; (e-5) the image of (e-4) with rotation restore; (e-6) the edge of the onelayer horizontal high-frequency image; (e-7) the edge of the one-layer vertical high-frequency image; (e-8) the edge of the one-layer diagonal high-frequency image; (e-9) the one-layer horizontal high-frequency image after the fusion; (e-10) the one-layer vertical high-frequency image after fusion; (e-11) the one-layer diagonal high-frequency image after fusion; (f-1) the denoised image using the method in this paper ( $\mathrm{db} 3$ wavelet); ( $\mathrm{f}-2)$ the denoising image using the method in this paper (sym3 wavelet); (g-1) the denoised image based on the traditional wavelet hard threshold (db3 wavelet); (g-2) the denoised image based on the traditional wavelet hard threshold (sym3 wavelet); (h-1) the denoised image based on the traditional wavelet soft threshold ( $\mathrm{db} 3$ wavelet); (h-2) the denoised image based on the traditional wavelet soft threshold (sym3 wavelet); (i-1) the denoised image using the method in [3] for hard thresholding (db3 wavelet); (i-2) the denoised image using the method in [3] for hard thresholding (sym3 wavelet); ( $\mathrm{j}-1)$ the denoised image using the method in [3] for soft thresholding (db3 wavelet); ( $\mathrm{j}-2$ ) the denoised image using the method in [3] for soft thresholding (sym3 wavelet); (k) the denoised image based on traditional SVD; (l) the denoised image using the method in this paper with the two-layer wavelet decomposition (db3 wavelet); (m) the denoised image using the method in this paper with the three-layer wavelet decomposition (db3 wavelet); (n) the PSNR results for different methods; (o) the PSNR results with 1 3-layer wavelet decomposition (db3 wavelet); (p-1) the PSNR with three denoising methods $\left(\sigma^{2}=0.001\right.$, db3 wavelet); (p-2) the PSNR with three denoising methods $\left(\sigma^{2}=0.001\right.$, sym3 wavelet); (q-1) the PSNR with three denoising methods $\left(\sigma^{2}=0.005\right.$, db3 wavelet); (q-2) the PSNR with three denoising methods ( $\sigma^{2}=0.005$, sym 3 wavelet); ( $\left.\mathrm{r}-1\right)$ the PSNR with three denoising methods $\left(\sigma^{2}=0.01\right.$, $\mathrm{db} 3$ wavelet); (r-2) the PSNR with three denoising methods $\left(\sigma^{2}=0.01\right.$, sym3 wavelet).

high-frequency information and using Roberts edge detection operator to detect the diagonal direction of the highfrequency information. Edge detection operators convolve with image through a series of windows with directions. These operators are $3 \times 3$ matrices. The following are the Sobel operators for edge detection in the horizontal and vertical directions, respectively:

$$
\begin{aligned}
& s=\left[\begin{array}{ccc}
-1 & -2 & -1 \\
1 & 0 & 0 \\
1 & 2 & 1
\end{array}\right], \\
& t=\left[\begin{array}{ccc}
-1 & 0 & 1 \\
-2 & 0 & 2 \\
-1 & 0 & 1
\end{array}\right],
\end{aligned}
$$

and the following are the Roberts operators for edge detection in $45^{\circ}$ and $135^{\circ}$ directions, respectively:

$$
\begin{aligned}
& s=\left[\begin{array}{ccc}
0 & 0 & 0 \\
0 & 1 & 0 \\
0 & 0 & -1
\end{array}\right], \\
& t=\left[\begin{array}{ccc}
0 & 0 & 0 \\
0 & 1 & 0 \\
-1 & 0 & 0
\end{array}\right] .
\end{aligned}
$$

Therefore, each operator can be used to match the two mutually perpendicular directions that are needed for different edge direction.

3.2. Determination of the Reconstruction of Singular Values. The number of the singular values used for the reconstruction 


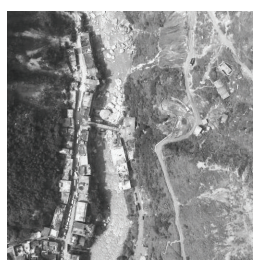

(a)

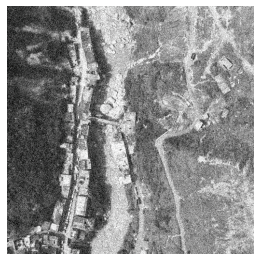

(e)

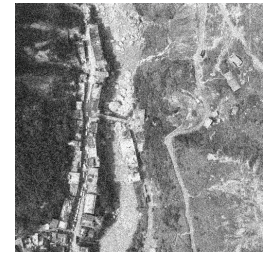

(b)

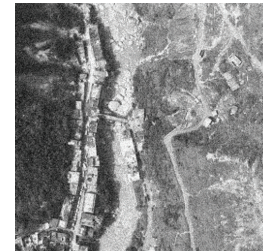

(f)

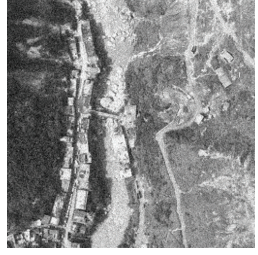

(c)

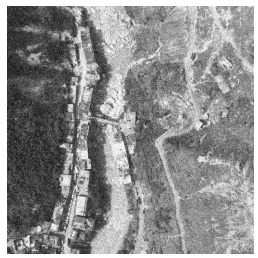

(g)

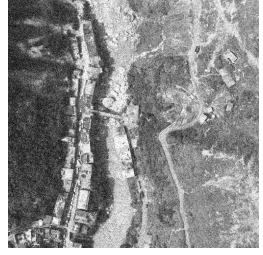

(d)

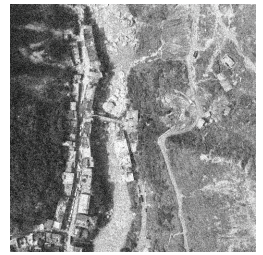

(h)

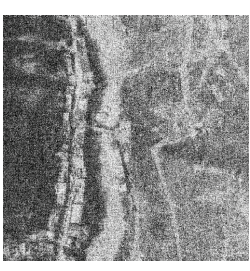

(i)

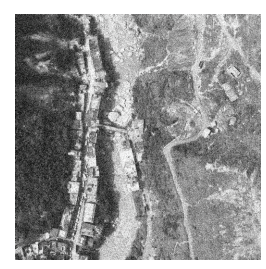

(j)

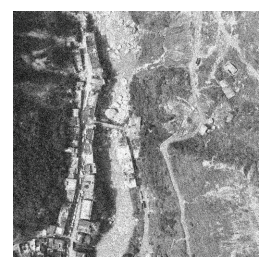

(k)

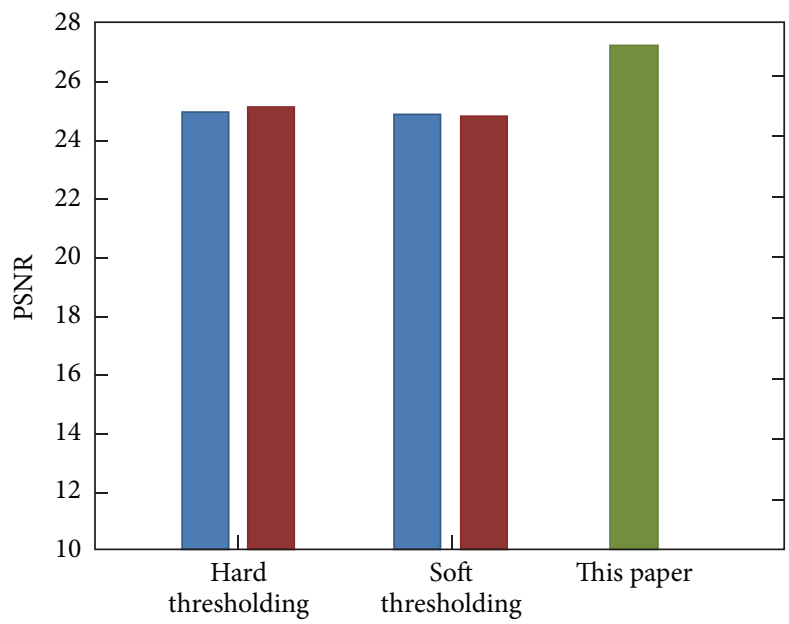

(l)

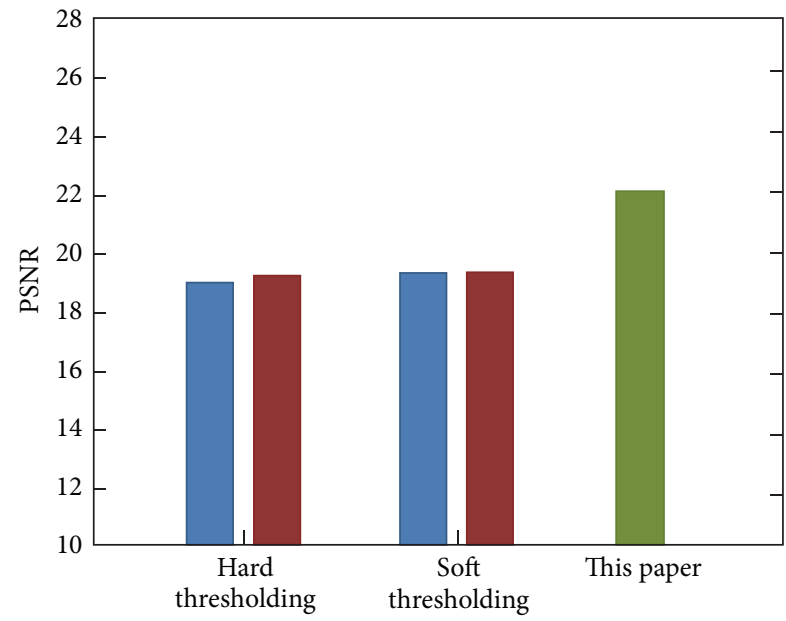

(n)

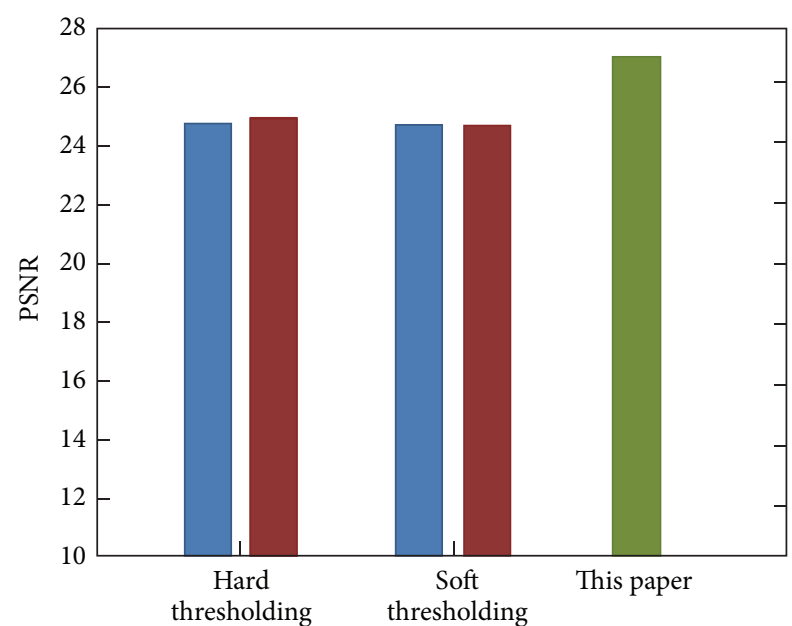

(m)

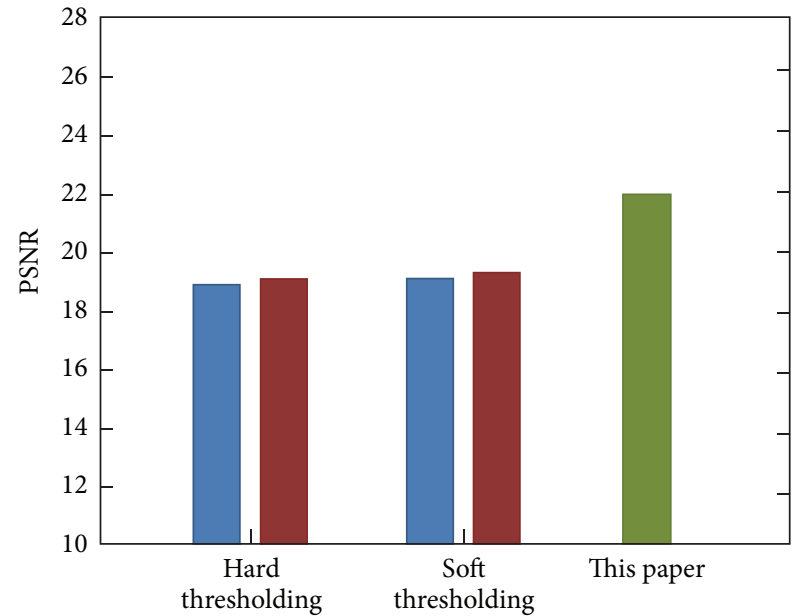

(o)

FIgure 5: Continued. 


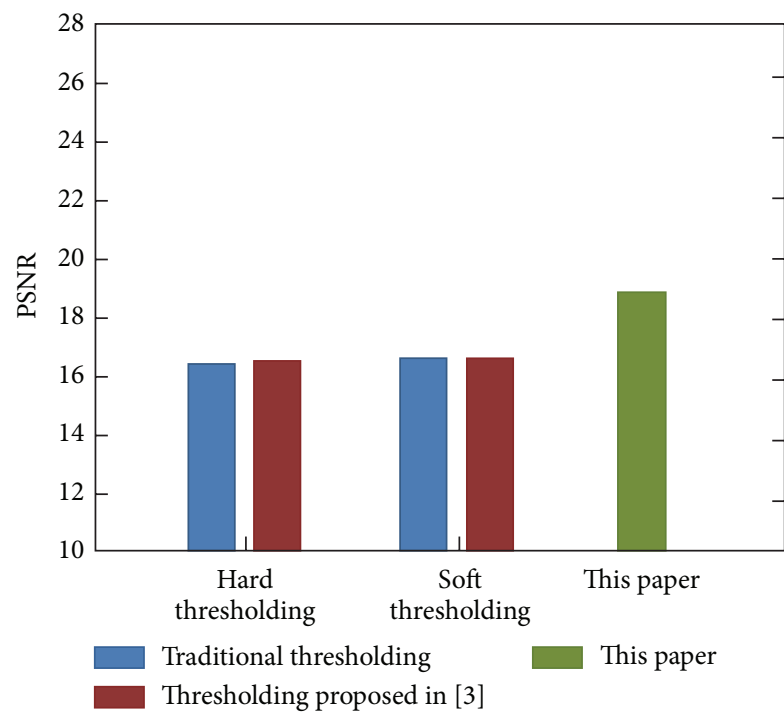

(p)

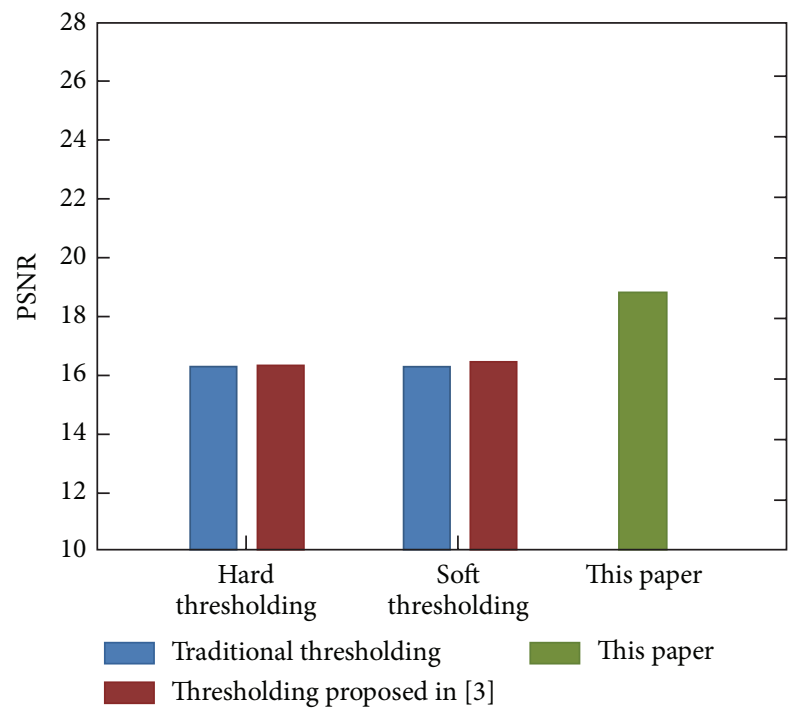

(q)

FIGURE 5: (a) The original image; (b) the noised image $\left(\sigma^{2}=0.01\right)$; (c) the denoised image using the method in this paper with the one-layer wavelet decomposition ( $\mathrm{db} 3$ wavelet); (d) the denoised image based on the traditional wavelet hard threshold (db3 wavelet); (e) the denoised image based on the traditional wavelet soft threshold ( $\mathrm{db} 3$ wavelet); (f) the denoised image using the method in this paper (sym3 wavelet); ( $\mathrm{g}$ ) the denoised image based on the traditional wavelet hard threshold (sym3 wavelet); (h) the denoised image based on the traditional wavelet soft threshold (sym3 wavelet); (i) the denoised image based on the traditional SVD; (j) the denoised image using the method in [3] for hard thresholding (db3 wavelet); (k) the denoised image using the method in [3] for soft thresholding (db3 wavelet); (l) the PSNR with three denoising methods $\left(\sigma^{2}=0.001\right.$, db3 wavelet); $(\mathrm{m})$ the PSNR with three denoising methods $\left(\sigma^{2}=0.001\right.$, sym 3 wavelet); (n) the PSNR with three denoising methods $\left(\sigma^{2}=0.005\right.$, db3 wavelet); (o) the PSNR with three denoising methods $\left(\sigma^{2}=0.005\right.$, sym 3 wavelet); (p) the PSNR with three denoising methods $\left(\sigma^{2}=0.01\right.$, db3 wavelet); (q) the PSNR with three denoising methods $\left(\sigma^{2}=0.01\right.$, sym 3 wavelet).

has $1 \sim R$ options. The traditional approach is to choose the singular value threshold $\varepsilon \leq \sqrt{l_{1} l_{2}} \sigma$; for Gauss white noise [4], the noise variance is $\sigma^{2}$. This formula gives the upper limit value only, but not the optimal threshold. This paper uses the greatest gradient of the PSNR as the measure criterion:

$$
\begin{aligned}
\operatorname{PSNR} & =10 \log _{10}\left(\frac{255^{2}}{\mathrm{MSE}}\right), \\
\mathrm{MSE} & =\frac{\sum_{1}^{M} \sum_{1}^{N}\left(f(i, j)-f_{0}(i, j)\right)^{2}}{M N}, \\
w(u) & =\frac{\operatorname{PSNR}(u+1)-\operatorname{PSNR}(u)}{k(u+1)-k(u)},
\end{aligned}
$$

where $f(i, j)$ is the pixel gray value of the denoising image, $f_{0}(i, j)$ is the pixel gray value of the original image, $k(u)$ is the total number of singular values, and $\operatorname{PSNR}(u)$ is the PSNR of the reconstructed image using the total number of the $u_{\text {th }}$ singular values.

\section{Simulations}

In the simulations, two images are used, to verify the validity of the method; the size of both images is $512 \times 512$, as shown in Figures 4(a) and 5(a). Figures 4(c) $-4(\mathrm{~m})$ are the denoised images for Figure 4(a).

For the one-layer wavelet decomposition, Figures 4(f-1)$4(\mathrm{k})$, respectively, illustrate the results of the following: (i) the image denoising method in this paper (with db3 and sym 3 wavelets in the wavelet transform, resp.);

(ii) the traditional wavelet hard threshold method (with $\mathrm{db} 3$ and sym 3 wavelets in the wavelet transform, resp.);

(iii) the traditional wavelet soft threshold method (with $\mathrm{db} 3$ and sym3 wavelets in the wavelet transform, resp.);

(iv) the hard thresholding method proposed in [3] (with $\mathrm{db} 3$ and sym 3 wavelets in the wavelet transform, resp.);

(v) the soft thresholding method proposed in [3] (with $\mathrm{db} 3$ and sym 3 wavelets in the wavelet transform, resp.);

(vi) the SVD filtering method.

The results of the image denoising method in this paper, using the 2 3-layer wavelet decomposition (with $\mathrm{db} 3$ wavelet), are obtained in Figures 4(1) and 4(m).

For Figure 5(a), the results are shown in Figures 5(c)5(i), using the method of this paper; as a comparison, Figures $5(\mathrm{j})$ and $5(\mathrm{k})$ are the results using the traditional wavelet hard threshold and soft threshold methods and the method proposed in the literature [3]. 


\section{Conclusions}

This paper provides an image denoising method, using the wavelet transform and the singular value decomposition (SVD), with the enhancement of the directional features. The experiments show that this method is effective in denoising, while it retains the original edge details, compared with the results using the relevant methods, and it also has a better performance than the recent wavelet threshold denoising method in [3].

\section{Conflict of Interests}

The authors declare that there is no conflict of interests regarding the publication of this paper.

\section{References}

[1] M. K. Mihçak, I. Kozintsev, K. Ramchandran, and P. Moulin, "Low-complexity image denoising based on statistical modeling of wavelet coefficients," IEEE Signal Processing Letters, vol. 6, no. 12, pp. 300-303, 1999.

[2] J.-L. Starck, E. J. Candès, and D. L. Donoho, "The curvelet transform for image denoising," IEEE Transactions on Image Processing, vol. 11, no. 6, pp. 670-684, 2002.

[3] C. He, J. Xing, J. Li, Q. Yang, and R. Wang, "A new wavelet threshold determination method considering interscale correlation in signal denoising," Mathematical Problems in Engineering, vol. 2015, Article ID 280251, 9 pages, 2015.

[4] R. Yang and M. Ren, "Wavelet denoising using principal component analysis," Expert Systems with Applications, vol. 38, no. 1, pp. 1073-1076, 2011.

[5] A. Fathi and A. R. Naghsh-Nilchi, "Efficient image denoising method based on a new adaptive wavelet packet thresholding function," IEEE Transactions on Image Processing, vol. 21, no. 9, pp. 3981-3990, 2012.

[6] L.-K. Shark and C. Yu, "Denoising by optimal fuzzy thresholding in wavelet domain," Electronics Letters, vol. 36, no. 6, pp. 581$582,2000$.

[7] T.-H. Yi, H.-N. Li, and X.-Y. Zhao, "Noise smoothing for structural vibration test signals using an improved wavelet thresholding technique," Sensors, vol. 12, no. 8, pp. 11205-11220, 2012.

[8] F. Autin, J.-M. Freyermuth, and R. von Sachs, "Combining thresholding rules: a new way to improve the performance of wavelet estimators," Journal of Nonparametric Statistics, vol. 24, no. 4, pp. 905-922, 2012.

[9] N. Sae-Bae and S. Udomhunsakul, "Adaptive block-based singular value decomposition filtering," in Proceedings of the Computer Graphics, Imaging and Visualisation (CGIV '07), pp. 298-303, IEEE, Bangkok, Thailand, August 2007. 


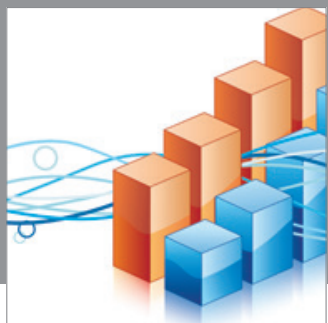

Advances in

Operations Research

mansans

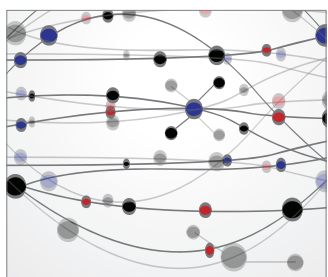

The Scientific World Journal
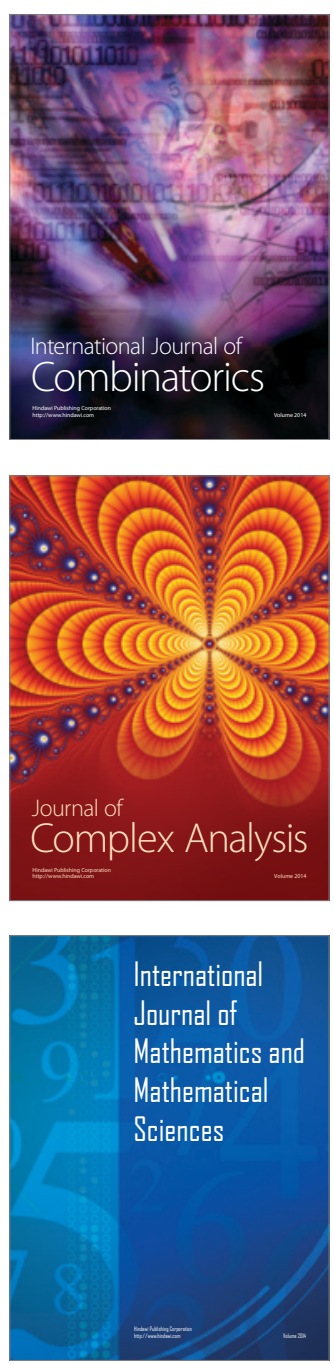
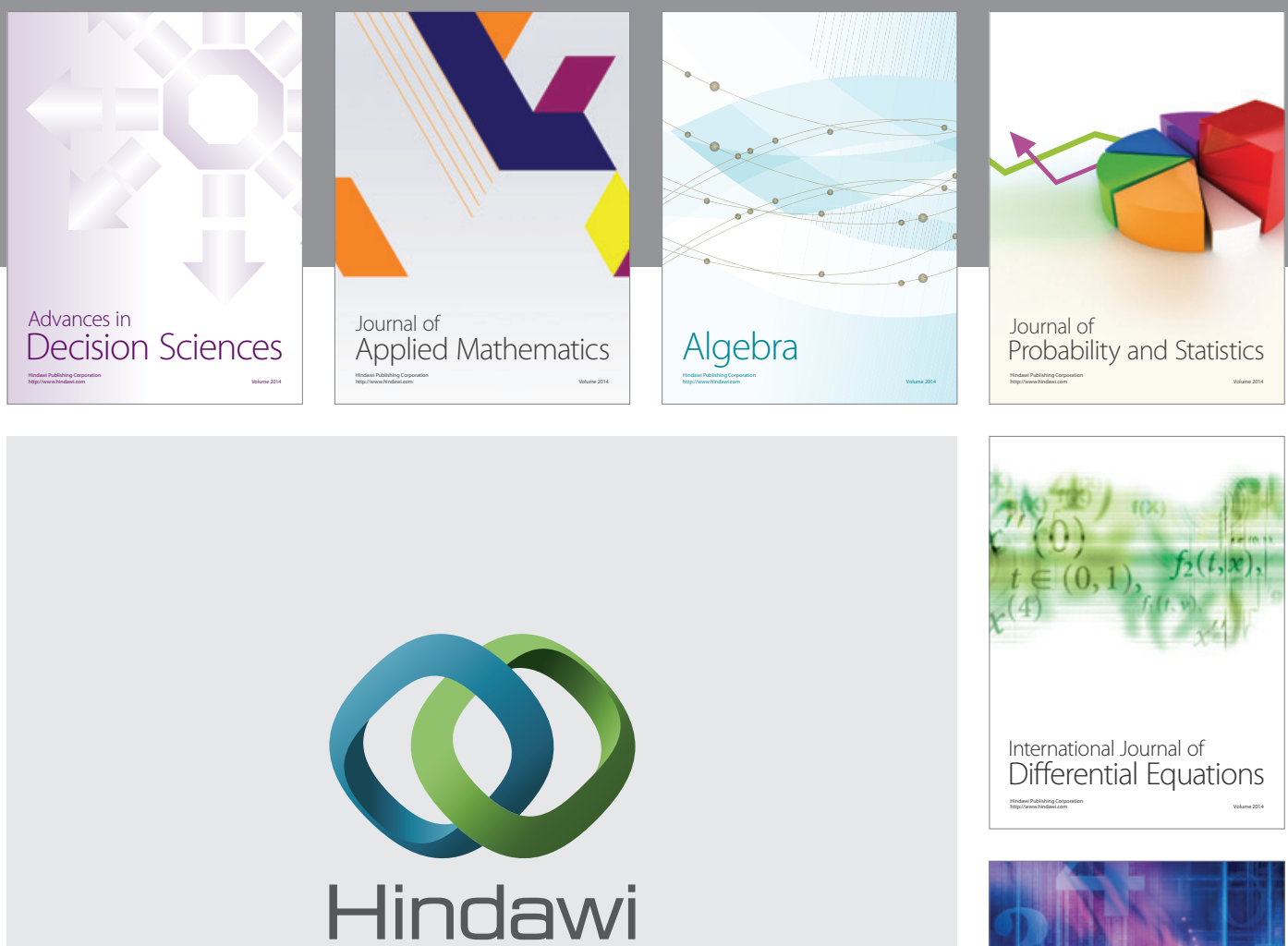

Submit your manuscripts at http://www.hindawi.com
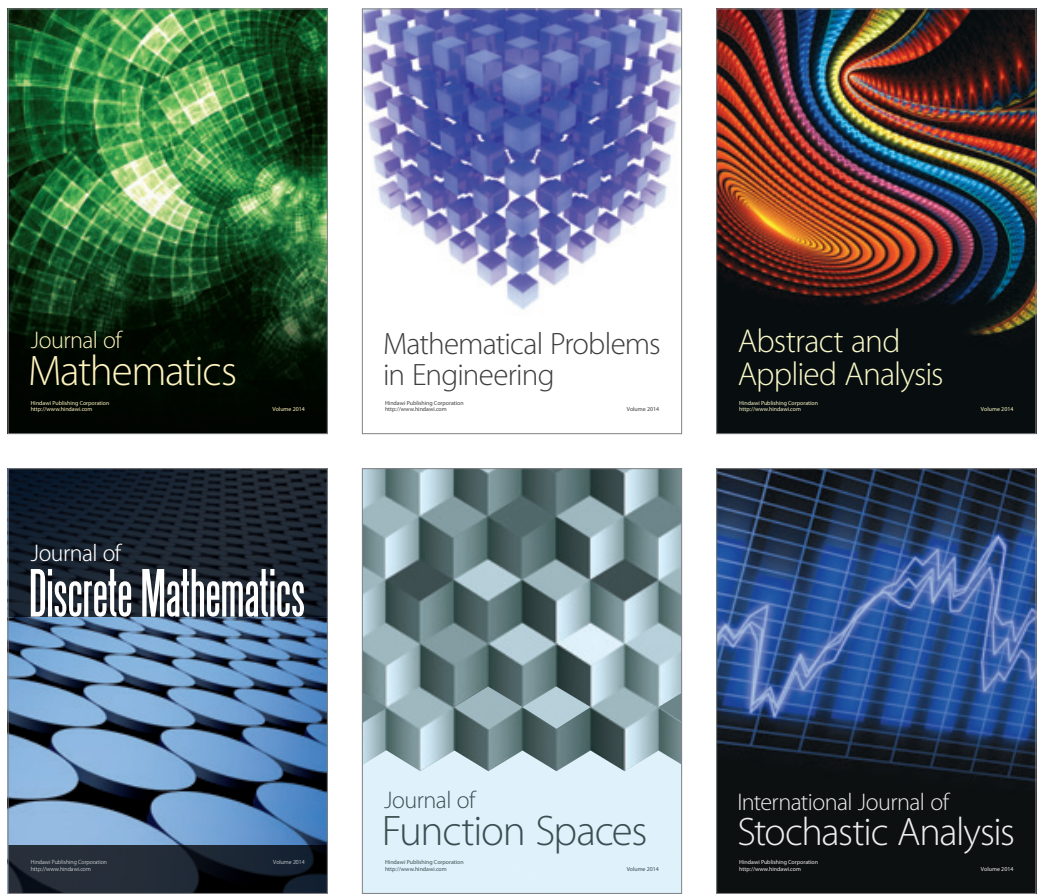

Journal of

Function Spaces

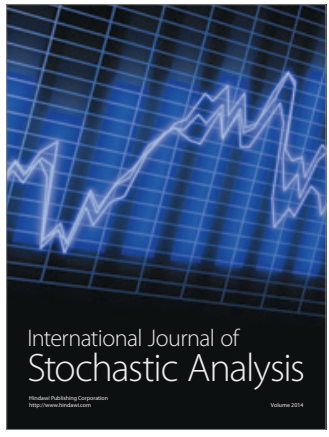

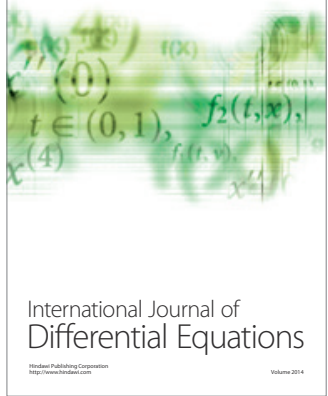
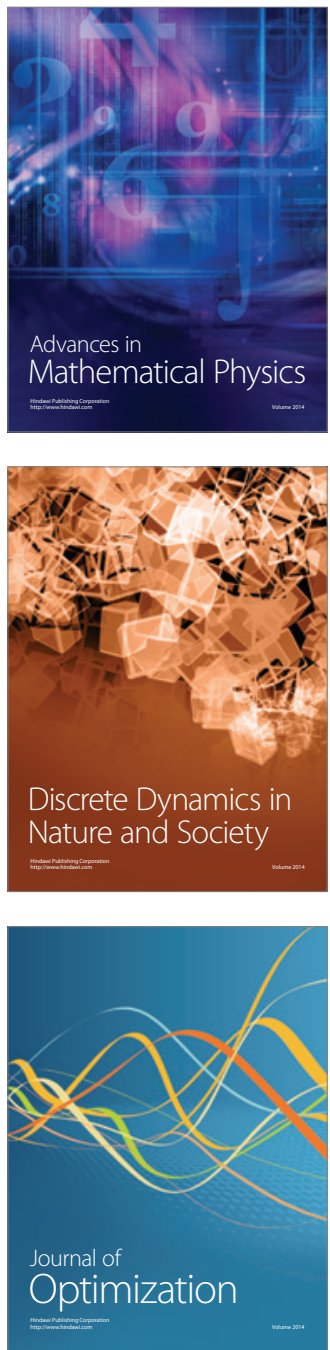